\title{
HAY YIELD AND QUALITY OF INTERCROPPED SORGHUM-SUDAN GRASS HYBRID AND LEGUMES WITH DIFFERENT SEED RATIO
}

\author{
Ugur BASARAN ${ }^{1}$, Medine COPUR DOGRUSOZ ${ }^{1}$,Erdem GULUMSER ${ }^{l}$, Hanife MUT ${ }^{l}$ \\ ${ }^{1}$ Bozok University, Faculty of Agriculture, Department of Field Crops, Yozgat, TURKEY \\ *Corresponding author: ugur.basaran@bozok.edu.tr
}

Received: 27.09.2016

\begin{abstract}
In this study, sorghum-sudangrass hybrid 'Aneto' and legumes (soybean and cowpea) intercropping at different seed rates (100:100; 50:100, 100:50) were examined for hay yield, protein content, protein yield,

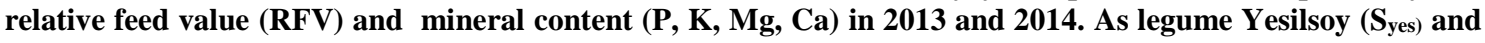
Yemsoy $\left(S_{y e m}\right)$ verieties of soybean and Ulkem $(C)$ varietiy of cowpea were used. Sorghum-sudangrass hybrid $x$ legume intercropping produced higher hay and protein yield compare to their monocrops. RFV of hay also exalted with intercropping copmpare to alone sorghum-sudangrass hybrid (S). Over the years, the highest hay yield was obtained from $S$ intercropped with $S_{\text {yes }}$ at 100:100 $\left(21.61 \mathrm{tha}^{-1}\right), 100: 50\left(20.68 \mathrm{t} \mathrm{ha}^{-1}\right)$ seed rates and

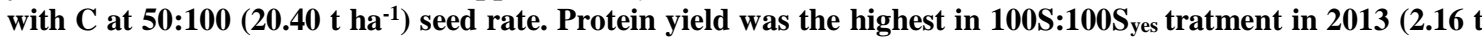
$\left.\mathrm{ha}^{-1}\right), 2014\left(2.85 \mathrm{t} \mathrm{ha}^{-1}\right)$ and combined years $\left(2.50 \mathrm{t} \mathrm{ha}^{-1}\right)$. However, $S$ intercropping with $\mathrm{C}$ at 100:100, 50:100

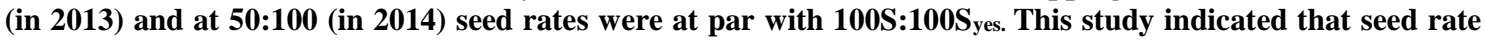
and species or variety selection extremly important in mixture cropping. Thus, generally sorghum-sudangrass hybrid and 'Yesilsoy' variety of soybean at 100:100 seed rate exhibited the best results in regarding hay and protein yield in the present conditions.
\end{abstract}

Key words: cowpea, hay yield, intercropping, sorghum-sudangrass hybrid, soybean,

\section{INTRODUCTION}

Dwindling soil and water resources and growing food demand require more efficient and sustainable production systems in agriculture. Intercropping is cultivating at least two crops in the same field simultaneously, may be result in increase of productivity in per unit area through efficient use of solar energy and optimization of soil and water resource (Hamd Alla et al., 2014). Intercropping might positively impact on sustainability through controlling weeds, insects, diseases (Egbe, 2005) and reducing water losses, soil erosion, nutrient leaching and $\mathrm{N}$ input (Ouma and Jeruto, 2010).

In forage production systems, the common type of intercropping is consisting of anuual crops and mostly cereal-legume combination (Francis, 1989). Cereals are important component of animal feeding with their high dry matter production. However, cereals forage is poor regarding nutritive value due to low protein content (Eskandari, et al., 2009). Legumes are good source of protein (Ayan et al., 2012). So, cereal protein shortage can be compensating by intercropping with legumes (Gebrehiwot et al., 1996) and, acceptable forage yield and quality can be possible. Intercropping is also very important for silage quality. All forages are not suitable for ensiling; especially pure legumes have poor fermentation quality (Ojeda, 2000). This constraint can be eliminated or mitigated with cereal-legume combination (Lima-Orozco et al., 2012).

Benefits in cereal-legume intercropping mainly depends on choose of the right crop combination and their proportion in the mixture (Singh et al., 2008, Asci et al., 2015). Plant density, different growing habits, shading and nutrition competition between plants could reduce the yield of mixtures (Seran and Brintha, 2010).

Sorghum $\mathrm{x}$ sudangrass hybrid (Sorghum bicolor $\mathrm{x}$ Sorghum bicolor var. sudanense) is a summer annual, high yielding, rapid growing and drought tolerant forage crop (Fribourg 1995) and can reach $3.7 \mathrm{~m}$ high (Ball et al., 2007). It is taller, coarser and more yielding than sudangrass. Less leaf area, secondary roots and waxy leaf surface makes sorghum-sudangrass more resistant to drought (Sarrantonio, 1994). Sorghum-sudangrass hybrids can produce more forage yield than maize under high temperature and drought (Uzun et al., 2009). Its' yield and quality more or less similar sorghum (Sorghum bicolor L.) (Ngongoni et al., 2007). Sorghum fodder is poor in quality due to low protein content (Ahmad et al., 2007). Legumes contain more than double of crude protein than forage sorghum, therefore, sorghum-legume intercropping has the potential to increase the biomass and quality of 
forage for per area compare to sole sorghum (Eskendari et al., 2009). Forage sorghum can be intercropped with forage legumes such as cowpea cluster bean, soybean etc. which are totally compatible with sorghum in terms of sowing time and irrigation (Iqbal et al., 2015).

In Turkey Sorghum and Sorghum $\mathrm{x}$ Sudangrass hybrids have gained attention for summer forage in irrigated areas and, it is mostly grown as alone for silage. Forage performance of sorghum or sorghum $\mathrm{x}$ sudangrass hybrids under sole cropping was reported in different region of Turkey (Cecen et al., 2005; Geren and Kavut., 2009; Uzun et al., 2009; Nazl1 et al., 2014; Karadag and Ozturk, 2014). However, any systematic study has not been performed so far to explore the possibilities of sorgum-legume intercropping. For this reason, there is a need to develop an appropriate sorgum- legume combination for higher yielding and quality forage production.

The present study was, therefore, aimed to explore the potential of forage sorghum (Sorghum $x$ Sudangrass hybrid)-legume intercropping systems with different seed ratio and the effects on yield and chemical composition of fodder in the irrigated conditions in Yozgat-Turkey.

\section{MATERIALS AND METHODS}

This study was performed during summer seasons of 2013 and 2014 in Research Field, Faculty of Agriculture, Bozok University located in Yerkoy-Yozgat. General soil characteristics of experimental area as fallows; clay-loam with low organic matter $(1.91 \%)$ and high $\mathrm{pH}(8.20)$, phosphorus content $8.62 \mathrm{~kg} / \mathrm{da}$, potassium content 48.47 $\mathrm{kg} / \mathrm{da}$. Long-term annual rainfall and main temperature during vegetation period (may-august) were $131.4 \mathrm{~mm}$ and $17.6{ }^{\circ} \mathrm{C}$. Average temperature and total rainfall in 2013 and 2014 growing seasons were $18.1^{\circ} \mathrm{C}, 61.3 \mathrm{~mm}$ and $18.5^{\circ} \mathrm{C}, 231.9 \mathrm{~mm}$, respectively.

Plant materials consisted of Aneto variety of sorghumsudangrass hybrid (Sorghum bicolor x S. bicolor var. sudanense), Yesilsoy $\left(\mathrm{S}_{\mathrm{yes}}\right)$ and Yemsoy $\left(\mathrm{S}_{\mathrm{yem}}\right)$ varieties of forage soybean (Glycine max L.) and Ulkem (C) variety of cowpea (Vigna unguiculata L.) were sown as binary mixtures with three seed rates $(100: 100,100: 50,50: 100)$ and as alone.

Field experiments were established on May 24, 2013 and May 13, 2014. Seed rate of each plant was determined based on alone sowing rate $(2.5 \mathrm{~kg} / \mathrm{da}$ for sorghumsudangrass hybrid, 20.000 plant $/ \mathrm{m}^{2}$ for soybean and cowpea). Row distance was arranged in $70 \mathrm{~cm}$ in alone plots. In mixtures, plants were sown in alternate rows with 35 distances. Plot area was $16.8 \mathrm{~m}^{2}$ (6 $\mathrm{m}$ long and 8 rows in mixtures; $6 \mathrm{~m}$ long and 4 rows in alone sowing). Experiments were arranged in a randomized complete block design with three replicates. As fertilizer; $3 \mathrm{~kg} / \mathrm{da} \mathrm{N}$ and $8 \mathrm{~kg} / \mathrm{da} \mathrm{P}_{2} \mathrm{O}_{5}$ were applied after planting. Additionally $3 \mathrm{~kg} / \mathrm{da} \mathrm{N}$ was applied when plants reach to $35 \mathrm{~cm}$ height. During the vegetation period, all the plots were irrigated three times until reached field capacity.
Mixture plots were harvested based on maturity of sorghum x sudangrass hybrid (when it was at dough stage) while alone legumes were at the end of seed filling. Plant height was performed just before the harvest. For fodder yield plant samples were dried $65{ }^{\circ} \mathrm{C}$ until constant weight. Crude protein (CP), acid detergent fiber (ADF), neutral detergent fiber (NDF), $\mathrm{Ca}, \mathrm{K}, \mathrm{Mg}$ and $\mathrm{P}$ content of hay was determined by using Near Reflectance Spectroscopy (NIRS, 'Foss 6500') with software package program 'IC-0904FE'.

Mean hay quality of mixtures was determined as fallows:

$$
\text { Mean Hay Quality }=\frac{\left(\mathrm{Y}_{\mathrm{C}} * \mathrm{X}_{\mathrm{C}} \%\right)+\left(\mathrm{Y}_{\mathrm{S}} * \mathrm{X}_{\mathrm{S}} \%\right)}{\Sigma \text { Yield }}
$$

Quality traits: CP (crude protein), ADF (Acid Detergent Fiber), NDF (Neutral Detergent Fiber) and mineral matters, Y: yield, S: sorghum-sudangrass hybrid, $\mathrm{C}$ : companion crop (soybean, cowpea), $\mathrm{X}$ : content of quality traits in forage.

Relative feed value (RFV) was estimated according to the following equations adapted from Rohweder et al. (1978);

$\mathrm{RFV}=(\% \mathrm{DDM} * \% \mathrm{DMI}) / 1.29 ; \% \mathrm{DDM}=88.9-$ $(0.779 \times \% \mathrm{ADF}) ; \mathrm{DMI} \%$ of $\mathrm{BW}=120 / \% \mathrm{NDF}$.

$\mathrm{DDM}=$ Dry matter digestibility, $\mathrm{ADF}=$ Acid detergent fibre $(\%$ of DM $), \mathrm{DMI}=$ Dry matter intake $(\%$ of BW)

The data was analyzed in separate and combined years. ANOVA was performed by using SPSS 13.0 package program (SPSS, Chicago, IL, USA) and, means were grouped with Duncan's multiple-range test.

\section{RESULTS}

\section{Morphological Development}

The effect of interceropping on plant height of sorghum $\mathrm{x}$ sudangrass hybrid (S) was significant in seperate and combined years (Table 1). Mean plant height of $\mathrm{S}$ and legumes were significantly different $(\mathrm{p} \leq 0.05)$ between years with higher values in 2014. Compare to alone sowing, intercropping with legumes significantly incresed height of $S(p \leq 0.05)$ in 2014. However, in 2013, the lowest height of $\mathrm{S}$ was determined in 100S:50S yes mixture and, majority of the sowing treatments were located in same group for $\mathrm{S}$ plant height. The effect of intercropping on legumes height was also significant $(\mathrm{p} \leq$ 0.05) (Table 1). However, this effect was held genotypedependent. Among sole legumes, Yesilsoy variety of soybean $\left(S_{y e s}\right)$ had the highest mean plant height $(1.16 \mathrm{~m})$ while Ulkem variety of cowpea (C) had the lowest height $(0.61 \mathrm{~m})$ and, this ranking was case for intercropping treatments. When legumes evaluated individually, seed rates were not much effective and, in terms of plant height, each genotype generally took place in same group under different seed rates. 
Table 1. Efect of intercropping on plant height of sorghum $\mathrm{x}$ sudangrass hybrid, soybean and cowpea in seperate and combined years

\begin{tabular}{|c|c|c|c|c|c|c|}
\hline \multirow{2}{*}{ Treatments } & \multicolumn{3}{|c|}{ Sorghum sudangrass hybrid (m) } & \multicolumn{3}{|c|}{ Legumes (m) } \\
\hline & $2013 *$ & $2014 *$ & Mean* & $2013 * *$ & $2014 * *$ & Mean* \\
\hline $100 \mathrm{~S}$ & $2.16 \mathrm{ab}$ & $2.09 \mathrm{~b}$ & $2.12 \mathrm{c}$ & - & - & - \\
\hline $100 \mathrm{~S}_{\text {yem }}$ & - & - & - & $0.86 \mathrm{bc}$ & $1.15 \mathrm{~b}$ & $0.96 \mathrm{bc}$ \\
\hline $100 \mathrm{~S}_{\text {yes }}$ & - & - & - & $1.09 \mathrm{a}$ & $1.29 \mathrm{ab}$ & $1.16 \mathrm{a}$ \\
\hline $100 \mathrm{C}$ & - & - & - & $0.52 \mathrm{gh}$ & $0.69 \mathrm{c}$ & $0.61 \mathrm{~d}$ \\
\hline $100 \mathrm{~S}: 100 \mathrm{~S}_{\mathrm{yem}}$ & $2.13 \mathrm{abc}$ & $2.79 \mathrm{a}$ & $2.46 \mathrm{ab}$ & $0.64 \mathrm{f}$ & $1.34 \mathrm{ab}$ & $0.99 \mathrm{~b}$ \\
\hline $100 \mathrm{~S}: 50 \mathrm{~S}_{\mathrm{yem}}$ & $1.81 \mathrm{c}$ & $2.75 \mathrm{a}$ & $2.28 \mathrm{bc}$ & $0.54 \mathrm{~g}$ & $1.13 \mathrm{~b}$ & $0.84 \mathrm{c}$ \\
\hline $50 \mathrm{~S}: 100 \mathrm{~S}_{\mathrm{yem}}$ & $2.13 \mathrm{abc}$ & $2.75 \mathrm{a}$ & $2.44 \mathrm{ab}$ & $0.66 \mathrm{ef}$ & $1.43 \mathrm{ab}$ & $1.04 \mathrm{ab}$ \\
\hline $100 \mathrm{~S}: 100 \mathrm{~S}_{\mathrm{yes}}$ & $2.31 \mathrm{a}$ & $2.80 \mathrm{a}$ & $2.56 \mathrm{a}$ & $0.74 \mathrm{de}$ & $1.56 \mathrm{a}$ & $1.15 \mathrm{a}$ \\
\hline $100 \mathrm{~S}: 50 \mathrm{~S}_{\mathrm{yes}}$ & $2.24 \mathrm{ab}$ & $2.65 \mathrm{a}$ & $2.45 \mathrm{ab}$ & $0.82 \mathrm{~cd}$ & $1.50 \mathrm{a}$ & $1.16 \mathrm{a}$ \\
\hline $50 \mathrm{~S}: 100 \mathrm{~S}_{\mathrm{yes}}$ & $2.29 \mathrm{a}$ & $2.47 \mathrm{a}$ & $2.38 \mathrm{ab}$ & $0.92 \mathrm{~b}$ & $1.41 \mathrm{ab}$ & $1.17 \mathrm{a}$ \\
\hline $100 \mathrm{~S}: 100 \mathrm{C}$ & $2.31 \mathrm{a}$ & $2.67 \mathrm{a}$ & $2.49 \mathrm{ab}$ & $0.44 \mathrm{~h}$ & $0.54 \mathrm{c}$ & $0.49 \mathrm{de}$ \\
\hline $100 \mathrm{~S}: 50 \mathrm{C}$ & $2.16 \mathrm{ab}$ & $2.70 \mathrm{a}$ & $2.43 \mathrm{ab}$ & $0.30_{1}$ & $0.55 \mathrm{c}$ & $0.43 \mathrm{e}$ \\
\hline $50 \mathrm{~S}: 100 \mathrm{C}$ & $1.94 \mathrm{bc}$ & $2.67 \mathrm{a}$ & $2.31 \mathrm{abc}$ & $0.59 \mathrm{fg}$ & $0.45 \mathrm{c}$ & $0.52 . \mathrm{de}$ \\
\hline Mean** & $2.14 B$ & $2.63 A$ & & $0.68 B$ & $1.23 \mathrm{~A}$ & \\
\hline
\end{tabular}

S: sorghum x sudangrass hybrid; $S_{\text {yem }}$ : Yemsoy variety of soybean; $S_{\text {yes }}$ : Yesilsoy variety of soybean; C: Ulkem variety of Cowpea

$*$ is significant at $P \leq 0.05, * *$ is significant at $P \leq 0.01$.

\section{Hay yield and quality of sole and intercropping}

As seen in Table 2, hay yield and crude protein content was significantly affected $(\mathrm{p} \leq 0.01)$ by cropping treatments and years. Over the treatments, mean hay yield and protein content were higher in 2014 than were in 2013. In regarding sole stand, S produced higher hay yield than legumes and, legumes took place in same group. Sole legumes produced also the lowest hay yield in saperate and combined years. Intercropping $\mathrm{S}$ with legumes significantly $(\mathrm{p} \leq 0.01)$ increased hay yield, except for with $S_{\text {yem }}$ at 100:100 and 100:50 seed rates in 2013 and at 100:50 seed rate in 2014. Over the years, the highest hay yield was obtained from $S$ intercropped with $S_{\text {yes }}$ at 100:100 (21.61 t ha-1), 100:50 (20.68 $\left.\mathrm{t} \mathrm{ha}^{-1}\right)$ seed rates and with $\mathrm{C}$ at 50:100 (20.40 $\left.\mathrm{t} \mathrm{ha}^{-1}\right)$ seed rate.

Table 2. Hay yield and crude protein content of sorghum x sudangrass hybrid-legume mixtures

\begin{tabular}{|c|c|c|c|c|c|c|}
\hline \multirow{2}{*}{ Treatments } & \multicolumn{3}{|c|}{ Hay Yield $\left(t\right.$ ha $\left.^{-1}\right)$} & \multicolumn{3}{|c|}{ Crude Protein Ratio (\%) } \\
\hline & $2013 * *$ & $2014 * *$ & Mean** & $2013 * *$ & $2014 * *$ & Mean** \\
\hline $100 \mathrm{~S}$ & $16.24 \mathrm{~b}$ & $18.15 \mathrm{de}$ & $17.19 \mathrm{e}$ & $7.88 \mathrm{e}$ & $8.45 \mathrm{e}$ & $8.16 \mathrm{~g}$ \\
\hline $100 \mathrm{~S}_{\mathrm{yem}}$ & $2.84 \mathrm{c}$ & $2.55 \mathrm{f}$ & $2.69 \mathrm{f}$ & $13.66 \mathrm{~b}$ & $10.61 \mathrm{~d}$ & $12.14 \mathrm{bc}$ \\
\hline $100 \mathrm{~S}_{\text {yes }}$ & $3.11 \mathrm{c}$ & $2.71 \mathrm{f}$ & $2.91 \mathrm{f}$ & $14.94 \mathrm{a}$ & $10.55 \mathrm{~d}$ & $12.75 \mathrm{~b}$ \\
\hline $100 \mathrm{C}$ & $3.18 \mathrm{c}$ & $1.75 \mathrm{f}$ & $2.46 \mathrm{f}$ & $15.65 \mathrm{a}$ & $14.23 \mathrm{a}$ & $14.94 \mathrm{a}$ \\
\hline $100 \mathrm{~S}: 100 \mathrm{~S}_{\mathrm{yem}}$ & $16.15 \mathrm{~b}$ & $19.79 \mathrm{~cd}$ & $17.97 \mathrm{de}$ & $8.15 \mathrm{e}$ & $11.85 \mathrm{bcd}$ & $9.99 \mathrm{f}$ \\
\hline $100 \mathrm{~S}: 50 \mathrm{~S}_{\mathrm{yem}}$ & $16.48 \mathrm{~b}$ & $17.59 \mathrm{e}$ & $17.04 \mathrm{e}$ & $8.95 \mathrm{de}$ & $11.45 \mathrm{bcd}$ & $10.20 \mathrm{ef}$ \\
\hline $50 \mathrm{~S}: 100 \mathrm{~S}_{\mathrm{yem}}$ & $18.68 \mathrm{ab}$ & $20.40 \mathrm{bc}$ & $19.54 \mathrm{bc}$ & $9.89 \mathrm{~cd}$ & $11.69 \mathrm{bcd}$ & $10.79 \mathrm{def}$ \\
\hline $100 \mathrm{~S}: 100 \mathrm{~S}_{\mathrm{yes}}$ & $19.91 \mathrm{a}$ & $23.32 \mathrm{a}$ & $21.61 \mathrm{a}$ & $10.90 \mathrm{c}$ & $12.23 \mathrm{a}-\mathrm{d}$ & $11.57 \mathrm{bcd}$ \\
\hline $100 \mathrm{~S}: 50 \mathrm{~S}_{\mathrm{yes}}$ & $18.66 \mathrm{ab}$ & $22.69 \mathrm{a}$ & $20.68 \mathrm{ab}$ & $9.13 \mathrm{de}$ & $12.31 \mathrm{a}-\mathrm{d}$ & $10.72 \mathrm{def}$ \\
\hline $50 \mathrm{~S}: 100 \mathrm{~S}_{\mathrm{yes}}$ & $17.46 \mathrm{ab}$ & $19.65 \mathrm{~cd}$ & $18.55 \mathrm{cde}$ & $8.95 \mathrm{de}$ & $11.14 \mathrm{~cd}$ & $10.05 \mathrm{f}$ \\
\hline $100 \mathrm{~S}: 100 \mathrm{C}$ & $17.52 \mathrm{ab}$ & $22.51 \mathrm{a}$ & $20.01 \mathrm{bc}$ & $9.99 \mathrm{~cd}$ & $13.46 \mathrm{ab}$ & $11.73 \mathrm{bcd}$ \\
\hline $100 \mathrm{~S}: 50 \mathrm{C}$ & $18.26 \mathrm{ab}$ & $19.97 \mathrm{c}$ & $19.11 \mathrm{bcd}$ & $9.08 \mathrm{de}$ & $13.48 \mathrm{ab}$ & $11.28 \mathrm{cde}$ \\
\hline $50 \mathrm{~S}: 100 \mathrm{C}$ & $18.88 \mathrm{ab}$ & $21.93 \mathrm{ab}$ & $20.40 \mathrm{ab}$ & $9.67 \mathrm{~cd}$ & $12.95 \mathrm{abc}$ & $11.31 \mathrm{cde}$ \\
\hline Mean** & $14.41 B$ & $16.40 \mathrm{~A}$ & & $10.52 B$ & $11.87 \mathrm{~A}$ & \\
\hline
\end{tabular}

S: sorghum $x$ sudangrass hybrid; $S_{\text {yem }}$ : Yemsoy variety of soybean; $S_{\text {yes }}$ : Yesilsoy variety of soybean; C: Ulkem variety of Cowpea

$*$ is significant at $P \leq 0.05, * *$ is significant at $P \leq 0.01$.

Alone $\mathrm{S}$ had the lowest crude protein ratio amongst cropping treatments as $7.88 \%, 8.45 \%$ and $8.16 \%$ in 2013 , 2014 and combined years, respectively. Expect for 2014, alone legumes had high protein content. In 2014, interestingly, $\mathrm{SxS}_{\mathrm{yes}}$ and $\mathrm{SxS}_{\mathrm{yem}}$ mixtures at all the seed rates exhibited higher protein content than alone $S_{\text {yes }}$ and $\mathrm{S}_{\text {yem. }}$. The highest protein content was determined in alone $\mathrm{C}$ hay in $2013(15.65 \%), 2014(14.23 \%)$ and combined years $(14.94 \%)$. However, in regarding protein content, alone $S_{y e s}$ in 2013 and $\mathrm{SxC}$ intercropping at 100:100, 100:50 seed rates in 2014 were at par with alone C.

The effect of year and cropping treatments also was significant on protein yield and Relative Feeed Value (RFV) of hay (Table 3). As in protein content and dry matter yield, mean protein yield was higher in 2014 than 
2013 over the cropping tratments. Although its high protein content, alone legumes had lowest protein yield due to low hay yield. Therefore, alone S produced higher protein yield than alone legumes in all years. Except $\mathrm{SxS}_{\mathrm{yem}}$ intercropping at all the seed rates in 2013, protein yield increased with Sxlegume intercropping compared to their alone sowing. Protein yield was the highest in $100 \mathrm{~S}: 100 \mathrm{~S}_{\text {yes }}$ tratment in 2013

$\left(2.16 \mathrm{t} \mathrm{ha}^{-1}\right), 2014$ (2.85 $\left.\mathrm{t} \mathrm{ha}^{-1}\right)$ and combined years $\left(2.50 \mathrm{t} \mathrm{ha}^{-1}\right)$. However, $\mathrm{S}$ intercropping with $\mathrm{C}$ at 100:100, 50:100 (in 2013) and at 50:100 (in 2014) seed rates were at par with $100 \mathrm{~S}: 100 \mathrm{~S}_{\text {yes. }}$

Table 3. Protein yield and relative feed value of of sorghum $x$ sudangrass hybrid-legume mixtures

\begin{tabular}{|c|c|c|c|c|c|c|}
\hline \multirow{2}{*}{ Treatments } & \multicolumn{3}{|c|}{ Protein Yield $\left(\mathrm{t} \mathrm{ha}^{-1}\right)$} & \multicolumn{3}{|c|}{ Relative Feed Value } \\
\hline & $2013 * *$ & 2014** & Mean** & $2013 * *$ & $2014 * *$ & Mean** \\
\hline $100 \mathrm{~S}$ & $1.27 \mathrm{~d}$ & $1.53 \mathrm{e}$ & $1.40 \mathrm{f}$ & $79.90 \mathrm{~b}$ & $69.21 \mathrm{f}$ & $77.56 \mathrm{e}$ \\
\hline $100 \mathrm{~S}_{\mathrm{yem}}$ & $0.38 \mathrm{e}$ & $0.27 \mathrm{f}$ & $0.33 \mathrm{~g}$ & $139.05 \mathrm{a}$ & $94.02 \mathrm{ab}$ & $116.54 \mathrm{~b}$ \\
\hline $100 S_{\text {yes }}$ & $0.46 \mathrm{e}$ & $0.28 \mathrm{f}$ & $0.37 \mathrm{~g}$ & $152.02 \mathrm{a}$ & $100.48 \mathrm{a}$ & $126.25 \mathrm{a}$ \\
\hline $100 \mathrm{C}$ & $0.49 \mathrm{e}$ & $0.24 \mathrm{f}$ & $0.37 \mathrm{~g}$ & $136.44 \mathrm{a}$ & $94.91 \mathrm{ab}$ & $115.67 \mathrm{~b}$ \\
\hline $100 \mathrm{~S}: 100 \mathrm{~S}_{\text {yem }}$ & $1.31 \mathrm{~d}$ & $2.35 \mathrm{~cd}$ & $1.83 \mathrm{e}$ & $90.52 \mathrm{~b}$ & 81.84 cde & $86.18 \mathrm{~cd}$ \\
\hline $100 \mathrm{~S}: 50 \mathrm{~S}_{\mathrm{yem}}$ & $1.47 \mathrm{~d}$ & $2.01 \mathrm{~d}$ & $1.74 \mathrm{e}$ & $91.32 \mathrm{~b}$ & 80.74 cde & $86.03 \mathrm{~cd}$ \\
\hline $50 \mathrm{~S}: 100 \mathrm{~S}_{\mathrm{yem}}$ & $1.84 \mathrm{~b}$ & $2.39 \mathrm{bcd}$ & $2.11 \mathrm{~cd}$ & $84.82 \mathrm{~b}$ & 78.38 def & $81.60 \mathrm{cde}$ \\
\hline $100 \mathrm{~S}: 100 \mathrm{~S}_{\mathrm{yes}}$ & $2.16 \mathrm{a}$ & $2.85 \mathrm{ab}$ & $2.50 \mathrm{a}$ & $85.98 \mathrm{~b}$ & $90.39 \mathrm{abc}$ & $88.19 \mathrm{~cd}$ \\
\hline $100 \mathrm{~S}: 50 \mathrm{~S}_{\mathrm{yes}}$ & $1.69 \mathrm{bc}$ & $2.79 \mathrm{abc}$ & $2.24 \mathrm{abc}$ & $92.22 \mathrm{~b}$ & $88.60 \mathrm{bcd}$ & $90.41 \mathrm{c}$ \\
\hline $50 \mathrm{~S}: 100 \mathrm{~S}_{\mathrm{yes}}$ & $1.55 \mathrm{bc}$ & $2.18 \mathrm{~d}$ & $1.87 \mathrm{de}$ & $84.87 \mathrm{~b}$ & 70.44 ef & $77.65 \mathrm{de}$ \\
\hline $100 \mathrm{~S}: 100 \mathrm{C}$ & $1.75 \mathrm{bc}$ & $3.03 \mathrm{a}$ & $2.39 \mathrm{ab}$ & $86.45 b$ & $86.77 \mathrm{bcd}$ & $86.61 \mathrm{~cd}$ \\
\hline $100 \mathrm{~S}: 50 \mathrm{C}$ & $1.66 \mathrm{bc}$ & $2.69 \mathrm{abc}$ & $2.17 \mathrm{bc}$ & $85.26 \mathrm{~b}$ & $85.96 \mathrm{bcd}$ & $85.61 \mathrm{~cd}$ \\
\hline $50 \mathrm{~S}: 100 \mathrm{C}$ & $1.82 \mathrm{~b}$ & $2.83 \mathrm{ab}$ & $2.33 \mathrm{abc}$ & $85.55 \mathrm{~b}$ & $72.92 \mathrm{ef}$ & $79.24 \mathrm{de}$ \\
\hline Mean** & $1.37 \mathrm{~B}$ & $1.96 \mathrm{~A}$ & & $99.57 A$ & $84.21 B$ & \\
\hline
\end{tabular}

RFV was the highest in alone legumes especially in $\mathrm{S}_{\text {yes }}$ (Table 3). Alone $\mathrm{S}$ had the lowest RFV and, intercropping with legumes significantly incresed RFV of hay. Among mixtures, $\mathrm{SxS}_{\mathrm{yes}}$ intercropping generally had higher RFV, however, in 2013, all the intercroping treatments statistically were similar. Relative Feed Value reflects digestibility (from $\% \mathrm{ADF}$ ) and intake potential (from \% NDF). Therefore, as expected, alone legumes had the lowest ADF and NDF content while alone S had the highest and, Sxlegume intercropping decreased ADF and NDF content of hay compare to alone $\mathrm{S}$ (data not given).

Mineral matter content of hay including phosphorus $(\mathrm{P})$, potassium $(\mathrm{K})$, calcium $(\mathrm{Ca})$ and magnesium $(\mathrm{Mg})$ were significantly different among cropping treatments (with exceptions) and between years (Table 4 and 5). When years compered, mean $\mathrm{P}$ and $\mathrm{K}$ content was higher in 2014 while $\mathrm{Ca}$ and $\mathrm{Mg}$ were higher in 2013 over the cropping treatments. There were no differences among cropping treatments in regarding P and K content in 2013 (Table 4). In 2014 and combined years, the effect of treatments was significant $(\mathrm{P} \leq 0.01)$ on $\mathrm{P}$ content of hay and, $\mathrm{SxC}$ mixtures exhibited the highest $\mathrm{P}$ content; however, some treatments were at par with these mixtures. Soybean varieties had the lowest P content in 2014 and combined years. In terms of $\mathrm{K}$ content, the effect of cropping treatments was significant $(\mathrm{P} \leq 0.05)$ in 2014 with the highest rates in 50S:100Syes $(2.05 \%)$, 50S:100C (1.94\%) and alone S (1.91\%).
The highest content of $\mathrm{Ca}$ was determined in alone $\mathrm{C}$ in seperate and combined years (Table 5) and, except 2014, soybean varieties were at par with C. Ca content was same in alone $S$ and Sxlegume mixtures in 2013 and 2014, however, it was lower in alone $S$ than mixtures in combined years. As in calcium, the highest percentage of magnesium were determined in monocrop cowpea hay, respectively $0.50 \%, 0.55 \%, 0.53 \%$ in years (Table 5) and followed by soybean varieties. Alone $\mathrm{S}$ was detected in the low $\mathrm{Mg}$ content and, significant increase was determined in some intercropping treatments changing year to year.

\section{DISCUSSIONS}

\section{Morphological Development}

Plant height of the sorghum-sudangrass hybrid (S) was higher in mixtures than in its sole cropping. It is possibly was due to light competition between plants. High plant density prevents the passage of light especially to the leaves on the bottom. So higher plant height may be for reaching the light. Adeniyan et al., (2014) raported that higher plant density in maize/cassava intercrop caused to increase the maize plant height. Similarly Hamd Alla et al, (2014) found that intercropping maize was taller than that in sole maize and authors artibuted this result to competition of crops for light. 
Table 4. Phosphorus (P) and potassium (K) content of sorghum $\mathrm{x}$ sudangrass hybrid -legume mixtures.

\begin{tabular}{|c|c|c|c|c|c|c|}
\hline \multirow{2}{*}{ Treatments } & \multicolumn{3}{|c|}{$\mathbf{P}(\%)$} & \multicolumn{3}{|c|}{ K (\%) } \\
\hline & 2013 & 2014** & Mean** & 2013 & 2014* & Mean \\
\hline $100 \mathrm{~S}$ & 0.26 & $0.31 \mathrm{abc}$ & $0.29 \mathrm{ab}$ & 1.04 & $1.91 \mathrm{ab}$ & 1.48 \\
\hline $100 \mathrm{~S}_{\mathrm{yem}}$ & 0.27 & $0.25 \mathrm{~d}$ & $0.26 \mathrm{bc}$ & 0.87 & $1.58 \mathrm{bc}$ & 1.23 \\
\hline $100 \mathrm{~S}_{\mathrm{yes}}$ & 0.26 & $0.25 \mathrm{~d}$ & $0.25 \mathrm{c}$ & 1.37 & $1.69 \mathrm{abc}$ & 1.53 \\
\hline $100 \mathrm{C}$ & 0.29 & $0.31 \mathrm{abc}$ & $0.30 \mathrm{a}$ & 1.35 & $1.65 \mathrm{abc}$ & 1.49 \\
\hline $100 \mathrm{~S}: 100 \mathrm{~S}_{\mathrm{yem}}$ & 0.26 & $0.33 \mathrm{ab}$ & $0.30 \mathrm{a}$ & 0.96 & $1.64 \mathrm{abc}$ & 1.3 \\
\hline $100 \mathrm{~S}: 50 \mathrm{~S}_{\mathrm{yem}}$ & 0.27 & $0.29 \mathrm{c}$ & $0.28 \mathrm{abc}$ & 1.37 & $1.42 \mathrm{c}$ & 1.39 \\
\hline $50 \mathrm{~S}: 100 \mathrm{~S}_{\mathrm{yem}}$ & 0.27 & $0.34 \mathrm{ab}$ & $0.30 \mathrm{a}$ & 0.85 & $1.83 \mathrm{abc}$ & 1.34 \\
\hline $100 \mathrm{~S}: 100 \mathrm{~S}_{\mathrm{yes}}$ & 0.28 & $0.32 \mathrm{abc}$ & $0.30 \mathrm{a}$ & 1.70 & $1.42 \mathrm{c}$ & 1.56 \\
\hline $100 \mathrm{~S}: 50 \mathrm{~S}_{\mathrm{yes}}$ & 0.26 & $0.33 \mathrm{abc}$ & $0.29 \mathrm{ab}$ & 1.28 & $1.55 \mathrm{bc}$ & 1.42 \\
\hline $50 \mathrm{~S}: 100 \mathrm{~S}_{\mathrm{yes}}$ & 0.25 & $0.31 \mathrm{bc}$ & $0.28 \mathrm{abc}$ & 0.91 & $2.05 \mathrm{a}$ & 1.48 \\
\hline $100 \mathrm{~S}: 100 \mathrm{C}$ & 0.26 & $0.35 \mathrm{a}$ & $0.31 \mathrm{a}$ & 0.80 & $1.43 \mathrm{c}$ & 1.12 \\
\hline $100 \mathrm{~S}: 50 \mathrm{C}$ & 0.26 & $0.35 \mathrm{a}$ & $0.31 \mathrm{a}$ & 1.12 & $1.55 \mathrm{bc}$ & 1.34 \\
\hline $50 \mathrm{~S}: 100 \mathrm{C}$ & 0.26 & $0.35 \mathrm{a}$ & $0.31 \mathrm{a}$ & 1.24 & $1.94 \mathrm{ab}$ & 1.59 \\
\hline Mean** & $0.26 B$ & $0.32 A$ & & $1.14 \mathrm{~B}$ & $1.6 \mathrm{~A}$ & \\
\hline
\end{tabular}

Table 5. Calcium $(\mathrm{Ca})$ and Magnesium $(\mathrm{Mg})$ content of of sorghum $\mathrm{x}$ sudangrass hybrid-legume mixtures

\begin{tabular}{|c|c|c|c|c|c|c|}
\hline \multirow{2}{*}{ Treatments } & \multicolumn{3}{|c|}{$\mathrm{Ca}(\%)$} & \multicolumn{3}{|c|}{$\operatorname{Mg}(\%)$} \\
\hline & $2013 * *$ & $2014 * *$ & Mean* & $2013 * *$ & $2014 * *$ & Mean** \\
\hline $100 \mathrm{~S}$ & $0.49 \mathrm{~b}$ & $0.26 \mathrm{c}$ & $0.38 \mathrm{c}$ & $0.27 \mathrm{ef}$ & $0.15 \mathrm{ef}$ & $0.20 \mathrm{e}$ \\
\hline $100 \mathrm{~S}_{\text {yem }}$ & $1.41 \mathrm{a}$ & $1.10 \mathrm{~b}$ & $1.26 \mathrm{a}$ & $0.40 \mathrm{~b}$ & $0.28 \mathrm{c}$ & $0.34 \mathrm{~b}$ \\
\hline $100 \mathrm{~S}_{\mathrm{yes}}$ & $1.36 \mathrm{a}$ & $1.16 \mathrm{~b}$ & $1.26 \mathrm{a}$ & $0.37 \mathrm{bcd}$ & $0.37 \mathrm{~b}$ & $0.37 \mathrm{~b}$ \\
\hline $100 \mathrm{C}$ & $1.28 \mathrm{a}$ & $1.39 \mathrm{a}$ & $1.34 \mathrm{a}$ & $0.50 \mathrm{a}$ & $0.55 \mathrm{a}$ & $0.53 \mathrm{a}$ \\
\hline $100 \mathrm{~S}: 100 \mathrm{~S}_{\mathrm{yem}}$ & $0.54 \mathrm{~b}$ & $0.40 \mathrm{c}$ & $0.47 \mathrm{bc}$ & $0.24 \mathrm{f}$ & 0.22 cde & $0.23 \mathrm{cde}$ \\
\hline $100 \mathrm{~S}: 50 \mathrm{~S}_{\mathrm{yem}}$ & $0.56 \mathrm{~b}$ & $0.44 \mathrm{c}$ & $0.50 \mathrm{bc}$ & $0.25 \mathrm{ef}$ & $0.17 \mathrm{def}$ & $0.21 \mathrm{de}$ \\
\hline $50 \mathrm{~S}: 100 \mathrm{~S}_{\mathrm{yem}}$ & $0.60 \mathrm{~b}$ & $0.44 \mathrm{c}$ & $0.52 \mathrm{bc}$ & $0.29 \mathrm{def}$ & $0.18 \mathrm{def}$ & 0.24 cde \\
\hline $100 \mathrm{~S}: 100 \mathrm{~S}_{\mathrm{yes}}$ & $0.53 \mathrm{~b}$ & $0.45 \mathrm{c}$ & $0.49 \mathrm{bc}$ & $0.24 \mathrm{f}$ & $0.19 \mathrm{def}$ & $0.22 \mathrm{de}$ \\
\hline $100 \mathrm{~S}: 50 \mathrm{~S}_{\mathrm{yes}}$ & $0.60 \mathrm{~b}$ & $0.33 \mathrm{c}$ & $0.46 \mathrm{bc}$ & $0.32 \mathrm{~b}-\mathrm{f}$ & $0.16 \mathrm{def}$ & 0.24 cde \\
\hline $50 \mathrm{~S}: 100 \mathrm{~S}_{\mathrm{yes}}$ & $0.64 \mathrm{~b}$ & $0.38 \mathrm{c}$ & $0.51 \mathrm{bc}$ & $0.34 \mathrm{~b}-\mathrm{e}$ & $0.13 \mathrm{f}$ & 0.23 cde \\
\hline $100 \mathrm{~S}: 100 \mathrm{C}$ & $0.64 \mathrm{~b}$ & $0.49 \mathrm{c}$ & $0.57 \mathrm{~b}$ & $0.31 \mathrm{c}-\mathrm{f}$ & $0.19 \mathrm{def}$ & $0.25 \mathrm{cde}$ \\
\hline $100 \mathrm{~S}: 50 \mathrm{C}$ & $0.61 \mathrm{~b}$ & $0.47 \mathrm{c}$ & $0.54 \mathrm{bc}$ & $0.31 \mathrm{c}-\mathrm{f}$ & $0.23 \mathrm{~cd}$ & $0.27 \mathrm{~cd}$ \\
\hline $50 \mathrm{~S}: 100 \mathrm{C}$ & $0.68 \mathrm{~b}$ & $0.32 \mathrm{c}$ & $0.50 \mathrm{bc}$ & $0.38 \mathrm{bc}$ & $0.18 \mathrm{def}$ & $0.28 \mathrm{c}$ \\
\hline Mean** & $0.77 A$ & $0.59 B$ & & $0.32 A$ & $0.23 B$ & \\
\hline
\end{tabular}

$S_{\text {yes }}$ plants were taller than $S_{\text {yem }}$ in all cropping systems with some exceptions. $S_{y e m}$ and $S_{y e s}$ varieties of soybean had generally similar plant height in mixtures, which may refers to their competitiveness. Similar results reported by Muoneke et al., (2007). However, differently, the increse in soybean plant height under intercropping, especially in narrow arrangement, was repored by Ennin et al., (2002). Intercropping reduced or not changed the height of cowpea. It might be associated with depressive effects of $\mathrm{S}$ or competition between $\mathrm{S}$ and cowpea. Previously, it was reported that under light-limited condition, cowpea produced more leaf with large surface rather than erect growth (Terao et al., 1997). In contrast, Hamd Alla et al, (2014) found that cowpea intercroped with maize had significanly higher plant height compare to alone sowing. Therefore, the differences in plant height among legume species and varieties under crropping systems could be attributed to the inherent characteristics and interactions among crops and ecological conditions.

\section{Hay yield and quality of sole and intercropping}

According to the average results, sorghum-sudangrass (S) when intercropped with legumes cowpea and soybean exhibited greater hay yield, protein yield, protein ratio and RFV than was in alone sowing. But this increase in yield and quality was highly dependent with the legume species or varieties, seed rate and year. On the other hand Sxlegume intercropping produced significantly higher hay yield and protein yield compare to alone legumes but, lower protein ratio as expected. Hamd Alla et al, (2014) who were study the effects of cowpea-maize intercropping on yield reported that intercropped maize with cowpea, exhibited greater values of straw and grain yield but, fresh and dry forage yields of cowpea reduced when it intercropped with maize compare to sole cropping. Greater values of mean hay yield, protein yield and protein content were noted in 2014. This can be explained by the higher rainfall and main temperature in 2014 than that in 2013 during the vegetation period. 
The mixtures including Yesilsoy $\left(\mathrm{S}_{\mathrm{yes}}\right)$ variety of soybean generally produced higher hay and protein yield. The highest hay yield was determined when $S$ intercropped with $S_{\text {yes }}$ at 100:100 and 100:50 seed rates (Table 2). S x $S_{\text {yes }}$ mixture with the 100:100 seed rate also had the greatest protein yield in seperate and combined years (Table 3), in addition, the mixtures including high seed rate of cowpea $(100 \%)$ took place in same group with these treatments regarding both hay yield and Proten yield in combined years with exceptions. These findings show that genotype selection and sowing rate are extremely critical issues to achive high yield and quality in intercropping as reporded by many authors (Hamd alla et al., 2014; Seran and Brintha, 2010; Singh et al., 2008). Also these results suggest that high yield and quality in cereal-legume intercropping might be associated with high rate of plants especeially of legumes in mixture. Similar findings reported by Gnanbari et al, (2010) who determined that total LER (land equivalent ratio) for yield was higher in $100 \%$ cowpea $+100 \%$ mazie intercropping. Contrarily, it was reported that straw yields of both sorghum and cowpea in sorgum-cowpea intecrrops with different planting patterns were higher in sole cropping than in the intercropping (Oseni, 2010).

More commonly known, legumes have much higher protein content than grasses (Eskandari et al., 2009). So, as accepted, the protein ratio in sole legumes especially that in $\mathrm{C}$ was supperior than other cropping systems. Alone $\mathrm{S}$ was the lowest protein content in both separate and combined years (Table 2). Therefore Sxlegume intercroping significantly increased protein content of hay compare to monocrop S. According to combined years, the highest crude protein content of hay was recorded for SxC mixture (11.73\%) at 100:100 seed rate and it was statistically at par with $\mathrm{SxS}_{\text {yes }}$ mixture (11.57\%) at 100:100 seed rate. The possitive effect of interceropping with legumes on protein content of cereals was previously reported by Ahmad et al, (2007).

In the present study alone legumes had the highest RFV while alone $\mathrm{S}$ had lowest RFV. So, as expected, Sxlegume mixtures exhibited higher RFV compere to alone S. Van Soest (1996) reported that the RFV is not a direct measure of the nutritional content but it is important for estimating the value of the forage. Legumes generally produce higher quality forage than grasses due to less fiber, favor higher crude protein and intake (Albayrak and Ek1z, 2005). Therefore one of the benefits of legumes in mixtures is improvement of forage quality besides the higher yield.

Juknevičius and Sabienè (2007) reported that mineral element content in the plants depend on species and families of plant; leguminous plants accumulated more $\mathrm{Ca}$ and $\mathrm{Mg}$ than cereal or grasses (Poaceae). However $\mathrm{K}$ and $\mathrm{P}$ accumulation of the families studied have not found different by the same authors. Therefore, legume and grasses mixtures may optimize the mineral content and improve the nutritional value of forages. Two year average results showed that $\mathrm{S}$-legume intercropping increase $\mathrm{Mg}$ and $\mathrm{Ca}$ content of the hay compare to alone
$\mathrm{S}$, while it did not show significant effect on $\mathrm{P}$ and $\mathrm{K}$ content. And also cropping treatments showed significant differences between years in terms of mineral content. These differences may be attribute climatic conditions and the ratio of species in the mixture. The ratio of the species in the mixture mostly can not reflect the sowing rate due to the interaction between plants or between plants and ecological conditions including soil, rainfal, temperature etc.

In conclusion, the present study showed that intercropping sorghum-sudangrass hybrid with cowpea, and soybean improved the yield and quality of the hay compare to their monocrops. Also this study indicated that seed rate and species or variety selection extremly important in mixture cropping. Thus, in generally, sorghum-sudangrass hybrid and 'Yesilsoy' variety of soybean at 100:100 seed rate exhibited the best results in regarding hay and protein yield in the present conditions.

\section{LITERATURE CITED}

Adeniyan, O. N., O.A. Aluko, S.O. Olanipekun, J.O. Olasoji and V.O. Aduramigba-Modupe. 2014. Growth and yield performance of cassava/maize intercrop under different plant population density of maize. J. of Agricultural Sci. 6(8): 3540.

Ahmad, A., A. Riaz, M. Naeem and A. Tanveer. 2007. Performance of forage sorghum intercropped with forage legumes under different planting patterns. Pakistan J. Bot. 39(2): 431-439.

Albayrak, S. and H. Ekiz. 2005. An investigation on the establishment of artificial pasture under Ankara's ecological conditions. Turk J. Agric For. 29: 69-74.

Asci, O.O., Z. Acar and Y.K. Arıcı. 2015. Hay yield, quality traits and interspecies competition of forage pea-triticale mixtures harvested at different stages. Turkish Journal of Field Crops, 20(2):166-173.

Ayan, I., H. Mut, U. Basaran, Z. Acar, O. Onal Asci. 2012. Forage potential of cowpea (Vigna unguiculata L. Walp). Turkish Journal of Field Crops, 17(2):135-138.

Ball, D.M., C.S. Hoveland and G.D. Lacefield. 2007. Southern Forages. 4th ed. International Plant Nutrition Institute, Norcross, GA.

Cecen, S., M. Oten and C. Erdurmus. 2005. Assesment of Sorghum (Sorghum bicolor L.), Sudangrass (Sorghum sudanense L.) and Corn (Zea mays L.) as Second Crop in the Coastal Region of West Mediterranean Belt of Turkey. J. of Agriculture Faculty of Akdeniz Univ. 18(3): 337-341.

Egbe, O.M. 2005. Evaluation of some agronomic potentials of pigeonpea genotypes for intercropping with maize and sorghum in Southern Guinea Savanna. Ph.D. Thesis, University of Agriculture, Makurdi, Nigeria.

Ennin, S.A., M.D. Clegg and C.A. Francis. 2002. Resource utilisation in soybean/maize intercrops. African Crop Science J. 10(3): 251-261.

Eskandari, H., A. Ghanbari and A. Javanmard. 2009. Intercropping of cereals and legumes for forage production. Notulae Scientia Biologicae. 1:7-13.

Francis, C.A. 1989. Biological efficiencies in multiple cropping systems. Advance in Agronomy. 42:1-42.

Fribourg, H.A. 1995. Summer annual grasses. In An Introduction to Grassland Agriculture edited by Barnes, R.F., Miller, D.A. \& Nelson, C.J. Forages, Vol. I, Ames Iowa: Iowa State University Press. pp. 463-472. 
Gebrehiwot, L., R.L. McGrow and G. Assefu. 1996. Forage yield and quality profile of three annual legumes in the tropical highlands of Ethiopia. J. of Agriculture. 73:83-98.

Geren, H. and Y.T. Kavut. 2009. An investigation on comparison of Sorghum (Sorghum sp.) species with corn (Zea mays L.) grown under second crop production. J. of Agriculture Faculty of Ege Univ. 46(1):9-16.

Ghanbari, A., M. Dahmardeh, B.A. Siahsar and M. Ramroudi. 2010. Effect of maize (Zea mays L.) - cowpea (Vigna unguiculata L.) intercropping on light distribution, soil temperature and soil moisture in arid environment. J. of Food, Agriculture \& Environment. 8(1):102-108.

Hamd Alla, W.A., E.M. Shalaby, R.A. Dawood and A.A. Zohry. 2014. Effect of cowpea (Vigna sinensis L.) with maize (Zea mays L.) intercropping on yield and its components. Int. J. of Biological, Biomolecular, Agricultural, Food and Biotechnological Engineering, 8(11):1263-1269.

Iqbal, M.A., A. Iqbal, K. Ali, H. Ali, R.D. Khan, A. Ahmad, F. Nabeel and A. 2015. Integration of forage sorghum and byproducts of sugarcane and sugar beet industries for ruminant nutrition: A Review. Global Veterinaria. 14(5):752-760.

Juknevicius, S. and N. Sabiene. 2007. The content of mineral elements in some grasses and legumes. Ekologija. 53(1):4452.

Karadag, Y. and M. Ozturk. 2014. Effect of different row spacings on the yield and quality of silage sorghum (Sorghum bicolor (L).Moench) cultivars to be second crop grown. J. of Agricultural Faculty of Gaziosmanpasa University. 31(1): 19-24

Lima-Orozco, R.A., A. Castro-Algeria and V. Fievez. 2012. Ensiled sorghum and soybean as ruminant feed in the tropics, with emphasis on Cuba. Grass and Forage Science. 68: 20-32.

Muoneke, C.O., M.A.O. Ogwuche and B.A. Kalu. 2007. Effect of maize planting density on the performance of maize/soybean intercropping system in a guinea savanna agroecosystem. Afr. J. Agric. Res. 2: 667-677.

Nazl1, R.I., I. Inal, A. Kusvuran and V. Tans1. 2014. Effects of different organic materials on forage production from sorghum x .sudangrass hybrid (Sorghum bicolor x Sorghum bicolor var. sudanense). Turk J. of Agricultural and Natural Sci. Special Issue: 2: 2075-2082.

Ngongoni, N.T., M. Mwale, C. Mapiye, M.T. Moyo, H. Hamudikuwanda and M. Titterton. 2007. Evaluation of cereal-legume intercropped forages for smallholder dairy production in Zimbabwe. Livestock Research for Rural Development. 19:126-134.

Ojeda, F. 2000. Harvesting and ensiling techniques. In: Silage Making in the Tropics with Particular Emphasis on Smallholders, Rome: FAO, ISBN: 978-92-5-104500-8.

Ouma, G. and P. Jeruto. 2010. Sustainable horticultural crop production through intercropping: The case of fruits and vegetable crops: A review. Agric. Biol. J. North America. 1(5):1098-1105.

Rohweder, D.A., R. F. Barnes and N. Jorgeson. 1978. Proposed hay grading standards based on laboratory analyses for evaluating quality. J. Animal Sci. 47:747-759.

Sarrantonio, M. 1994. Northeast cover crop handbook. Rodale Institute, Emmaus, PA.

Seran, T.H. and I. Brintha. 2010. Review on maize based intercropping. J. of Agronomy. 9(3):135-145.

Singh, U., A.A. Saad and S.R. Singh. 2008. Production potential, biological feasibility and economic viability of maize (Zea mays L.) based intercropping system under rainfed conditions. Indian J. of Agricultural Sciences. 78(12):10231027.

Terao, T., I. Wantabe, R. Matsunaga, S. Hakoyame and B.B. Singh. 1997. Agrophysiological constraints in intercropped cowpea: an analysis. Pages 120- 140 in Advances in cowpea research, edited by B. B, Singh, D. R, Mohan Raj, K. E, Dashiel, \& L. E. N, Jackai. Copublication of IITA and JIRCAS, IITA, Ibadan, Nigeria.

Uzun, F., S. Ugur and M. Sulak. 2009. Yield, nutritional and chemical properties of some sorghum $\mathrm{x}$ sudangrass hybrids (Sorghum bicolor (L.) Moench $\mathrm{x}$ Sorghum sudanense Stapf.). Journal of Animal and Veterinary Advances. 8: 1602-1608.

Van Soest, P.J. 1996. Allometry and ecology of feeding behavior and digestive capacity in herbivores: A Review. Zoo Biology. 15:455-479. 